\title{
Pro-environmental behaviors correlate with delay
}

\section{discounting}

Shahabeddin Sahraeian ${ }^{1, *}$, Iman Khanahmadi ${ }^{2}$, and Hashem Sadeghiyeh ${ }^{3}$

${ }^{1}$ Faculty of Management, University of Tehran, Tehran, Iran

${ }^{2}$ Faculty of Law and Political Science, University of Tehran, Tehran, Iran

${ }^{3}$ Department of Psychology, University of Arizona, Tucson, Arizona, USA

Working Paper

*Corresponding author: shahab.sa@gmail.com

September 1, 2021 


\begin{abstract}
A total of 138 participants were recruited via Amazon Mechanical Turk to evaluate the relationship between temporal discounting and pro-environmental behavior. Pro-environmental behavior was assessed by using a modified version of Whitmarsh \& O'Neill [1] survey. Temporal discounting was assessed by Kirby's 27-item delay-discounting measure [2]. The results showed a positive trend correlation between pro-environmental behavior and temporal discounting; i.e. the more discounting of the future was correlated with less proenvironmental behavior. This trend became much more significant when we just analyzed those participants who answered "Yes" to the question "Do you think climate change is something that is affecting or is going to affect you, personally?" to which 98 out of 138 participants of our study answered "yes". For this upper $\% 70$ of participants the Pearson correlation coefficient was $r=0.28 ; p=0.006$. We also analyzed different demographic traits to see whether there is any difference between sub-groups regarding differential pro-environmental behaviors. Two features turned out to be highly predictive of the pro-environmental behavior: education and marriage. Married and higher educated tend to act more pro-environmentally than single and lower educated.
\end{abstract}

Keywords - temporal discounting, delay discounting, sustainability, pro environmental, green personality, global warming 


\section{Introduction}

World Commission on Environment and Development [3] defined sustainability as behavior "that meets the needs of the present without compromising the ability of future generations to meet their own needs". There is an urgent challenge to promote sustainable development in the environmental, economic and social / political landscape, since people around the world face many problems from poor water quality, and threats of natural disasters, to extreme poverty, and lack of access to well quality food, education and health-care system [4]. The real main cause of these sustainability issues are the effects of our species behavior. These effects include all of our actions, omissions, communications, and decision makings that directly or indirectly affect issues of environments and sustainability. These human behaviors include political decisions made by political leaders, production patterns and resource allocation by companies, and consumption patterns by consumers.

Previous studies on pro-environmental behaviour point to a variety of parameters that impact sustainable attitudes [5]. At an abstract level, it may seem that best predictor of pro-environmental behavior is "value", how much people care about the topic and what is the role of that topic in their view point of world [6, 7]. But the value is too abstract to practically approach. We need to tackle more concrete constructs like cognitive and emotional traits and personalities [8-11]. Many research tackle similar problems by pursuing structurally definable variables [12-19], Also, as noted by Whitmarsh and O'Neill [20], it is crucial to understand that to provoke pro-environmental behaviour, you don't need to have "environmental concern" or "pro-environmental values" at all [21]. In a study it was shown that the proportion of people who took actions directly out of concern for climate change were lower than the proportion of those who claim to conserve energy [22] Energy conservation is motivated much more by financial and health-related benefits than by pro-environmental "concern" or "value" [23].

In the current research, we aimed to investigate the relationship between delay discounting and proenvironmental behaviors using an individual differences approach. That means, we asked whether people with higher delay discounting trait show less or more pro-environmental behaviors. We used the 27-item Delay Discounting Questionnaire [2] to measure temporal discounting. In this questionnaire, participants choose between between small but immediate amounts of money and a larger but delayed amounts of money (e.g. $\$ 11$ now or $\$ 30$ in two weeks). Based on participants' pattern of choosing between immediate and delayed options, a parameter $k[24]$ is calculated for each participant which estimates their average discounting rate for delayed rewards. Delay discounting has long been proposed to be 
related to doing the immediate harder work for the benefit of the delayed more rewarding consequence. It is also a measure of how we trade off selfish actions versus public-oriented actions that ultimate benefit us (myopic versus well-thought selfishness actually). Pro-environmental care is a classic example of this "Tragedy of the commons" problem [25]. There have been many researches studying the relationship between delay discounting and pro-environmental behaviors [4, 26-31]. But no conclusive results has been obtained. This is partly due to the application of different measure and concepts. Here we apply some specific measures to assess delay discounting and the pro-environmental behaviors and then we discuss how this would compare to other research on the topic.

\section{Methods}

\section{Participants}

A total of hundred and thirty eight participants took part in our online Amazon Mechanical Turk experiment $(\mathrm{N}=138 ;$ Males $=89 ;$ Females $=49 ;$ ages $=20-70 ;$ mean $=37.64)$. After giving their consent on the first, participants answered the delay discounting questionnaire on page 2. All 27 questions were presented in a single page. Then they proceeded to answer the pro-environmental package in the following five pages. Participants were all residents of different states in the United States. Previous research [32, 33] showed that the participants recruited from Amazon Turk population could be more representative than participants sampled conveniently and frequently used in most psychological research. Therefore Amazon Turk data is at least as reliable as other ususual psychological experiments data from student samples [33-35]. All participants gave informed consent and the study was approved by the Institutional Review Board at the Missouri University of Science and Technology. All experiments were performed in accordance with relevant guidelines and regulations.

\section{Delay Discounting}

We used Kirby and Maraković’s 27-item questionnaire to assess temporal discounting [2, 36]. Each item inquired about choice between a monetary amount delivered immediately and a larger amount delivered after a delay. For example, the questionnaire asked, "Would you prefer $\$ 14$ today, or $\$ 25$ in 19 days?". The amount of smaller-immediate reward ("today" option), larger-delayed reward ("later" option) and the delay (in terms of days) vary in those 27 questions ("today" reward between $\$ 11$ - \$80; "later" reward 
between $\$ 25$ - \$85; Delay between 7 - 186 days). We used the complete 27 items in the order as follows:

1. Would you prefer $\$ 54$ today or $\$ 55$ in 117 days?

2. Would you prefer $\$ 55$ today or $\$ 75$ in 61 days?

3. Would you prefer $\$ 19$ today or $\$ 25$ in 53 days?

4. Would you prefer $\$ 31$ today or $\$ 85$ in 7 days?

5. Would you prefer $\$ 14$ today or $\$ 25$ in 19 days?

6. Would you prefer $\$ 47$ today or $\$ 50$ in 160 days?

7. Would you prefer $\$ 15$ today or $\$ 35$ in 13 days?

8. Would you prefer $\$ 25$ today or $\$ 60$ in 14 days?

9. Would you prefer $\$ 78$ today or $\$ 80$ in 162 days?

10. Would you prefer $\$ 40$ today or $\$ 55$ in 62 days?

11. Would you prefer $\$ 11$ today or $\$ 30$ in 7 days?

12. Would you prefer $\$ 67$ today or $\$ 75$ in 119 days?

13. Would you prefer $\$ 34$ today or $\$ 35$ in 186 days?

14. Would you prefer $\$ 27$ today or $\$ 50$ in 21 days?

15. Would you prefer $\$ 69$ today or $\$ 85$ in 91 days?

16. Would you prefer $\$ 49$ today or $\$ 60$ in 89 days?

17. Would you prefer $\$ 80$ today or $\$ 85$ in 157 days?

18. Would you prefer $\$ 24$ today or $\$ 35$ in 29 days?

19 . Would you prefer $\$ 33$ today or $\$ 80$ in 14 days?

20. Would you prefer $\$ 28$ today or $\$ 30$ in 179 days?

21 . Would you prefer $\$ 34$ today or $\$ 50$ in 30 days?

22 . Would you prefer $\$ 25$ today or $\$ 30$ in 80 days?

23. Would you prefer $\$ 41$ today or $\$ 75$ in 20 days?

24. Would you prefer $\$ 54$ today or $\$ 60$ in 111 days?

25 . Would you prefer $\$ 54$ today or $\$ 80$ in 30 days?

26. Would you prefer $\$ 22$ today or $\$ 25$ in 136 days?

27 . Would you prefer $\$ 20$ today or $\$ 55$ in 7 days?

Responses determined the degree to which participants discounted delayed rewards, by estimating $\mathrm{k}$ values obtained at three magnitudes of rewards contained within the questionnaire [37]. Prior studies of this questionnaire show that $\mathrm{k}$ values differ between substance abusers and controls in the expected 
manner, and the degree of delay discounting decreases as reward amount increases [36], consistent with other methods of assessing the steepness of the delay discounting curve [38]. Kirby and colleagues [2] detail methods for deriving $\mathrm{k}$ values from this questionnaire, and the approach is similar to those described for assessing $\mathrm{h}$ values from the probability discounting questionnaire [39].

To quantify delay discounting we used several different measures. The most straightforward one is to just count the number of options referring to now-today choice. By this measure, the greater delay discounting would be correlated with the larger number of "today" options.

However, more sophisticated measures of delay discounting could be obtained by model fitting. The widely used model is to fit a hyperbolic discount factor to the data. More specifically, we assume that the future reward, $A$, arriving after a delay $D$, is discounted according to a hyperbolic discount factor 40 , 41]:

$$
V=A /(1+k D)
$$

where $k$ is the discount factor (subject-specific). To fit $k$ we used the publicly-accessible spreadsheet provided by Kirby [42]. Their method is based on the method described by Kaplan and colleagues [24]. In addition to computing an overall $k$ factor by including all the twenty seven options, they also calculated several other discount factors for small, medium and large reward items. The idea is that temporal discounting might be different for different range of magnitudes of rewards. Also they computed the geometric mean of those k's (small, medium and large $k$ s). Based on the range of monetary values, the 27 choices are divided into three 9-item categories: small, medium and large ranges. Then, based on the hyperbolic discounting equation (Eq. 1), it finds a $\mathrm{k}$ value for each item as a point in which there is no difference between choosing "today" and "later" options for that item. Then for each participant based on his/her answers and the patterns of switches from "today" to "later" options and the reverse, it gives us a k-value for each 9-item category: Small k, Medium k, Large k.

For example, in question 2 it asks: Would you prefer $\$ 55$ today, or $\$ 75$ in 61 days? The indifference point is when the $\$ 75$ in 61 days worth as $\$ 55$ today. We can calculate the $\mathrm{k}$ for the indifference point, in which the "today" and "later" choices look the same, by plugging $V=55, A=75, D=61$ in Eq. (1):

$$
\begin{gathered}
55=75 /(1+61 * k) \\
k=((75 / 55)-1) / 61 \\
k=0.00596125
\end{gathered}
$$


If a participant choose "today" for this question, they have a $k>0.00596125$.

Similarly, if the same participant answer "later" in question 7: Would you prefer $\$ 15$ today, or $\$ 35$ in 13 days?, the indifference point would be $k=((35 / 15)-1) / 13=0.102564103$ so our participant would have a $k<0.102564103$. So for this participant given these two questions, we can estimate their $\mathrm{k}$ to be between $0.00596125<k<0.102564103$. By adding more questions, we can obtain better estimates for $k$.

Thus we have six measures of temporal discounting for each subject: the fraction of "today" choices, overall $k$, small $k$, medium $k$, large $k$, and the geometric mean of small, medium and large $k$ s.

\section{Pro-environmental Measures}

We assessed pro-envirromental behavior by a questionnaire adopted from a 2010 paper by Whitmarsh and colleagues and a 2013 paper by Markle and colleagues [1, 43].

Markle and colleagues developed a pro-environmental self-identity scale and a Pro-environmental behaviour (PEB) using measures adapted from previous research. [7, 21, 44,46]. There were 4 items for the self-identity:

'I think of myself as an environmentally-friendly consumer',

'I think of myself as someone who is very concerned with environmental issues',

'I would be embarrassed to be seen as having an environmentally- friendly lifestyle' (scoring reversed), and

'I would not want my family or friends to think of me as someone who is concerned about environmental issues' (scoring reversed).

These questions were assessed on a likert 5-point agreement scale. The internal consistency measure was reported satisfactory and reliable (alpha $=.7)$.

The PEB part included different multiple categories of actions and their frequencies and also the consequences of those behaviors [21]. In a new review in United Kingdom, which was conducted by DEFRA [45], they recognized twelve main behaviors (so called 'headline behaviours'). These headline behaviors encompassed both high and low pro-environmental actions. It also included regular and oneoff actions, corresponding to 4 behavioural aspects: domestic energy and water usage, waste decisions, transport acts, and ecologically friendly consumption / shopping:

1. "Domestic energy/water use includes: installing insulation products, better energy management and usage, installing domestic micro-generation through renewables, and more responsible water usage." 
2. "Waste behaviours include: increasing recycling and segrega- tion, and wasting less (food)."

3. "Transport actions include: buying/using more energy-efficient (low-carbon) vehicles, using the car less - seeking alternatives for short trips ( $<3$ miles), and reducing non-essential flying (short haul)."

4. "Shopping choices include: buying energy-efficient products, eating food locally in season, and adopting a diet with lower environmental impacts." [43].

\section{Questionnaires:}

Please indicate the last time you took this action (if at all): [Never, 5 or more years ago, 1-4 years ago, In the last year]

- Installed insulation products in your home

- Bought or built an energy-efficient home

- Installed a more efficient heating system

- Installed a renewable energy system (e.g., solar panels, wind turbine) in your home

- Changed to a 'green' energy tariff for your home

- Bought a low-emission vehicle (e.g., hybrid, electric, biofuel, less than 1.4 L engine)

- Bought a product to save water (e.g., water butt, water 'hippo', low-flush toilet)

Please indicate how often you take each action: [Never, Occasionally, Often, Always]

- Turn off lights you're not using

- Drive economically (e.g., braking or accelerating gently)

- Walk, cycle or take public transport for short journeys (i.e., trips of less than 3 miles)

- Use an alternative to travelling (e.g., shopping online)

- Share a car journey with someone else

- Cut down on the amount you fly

- Buy environmentally-friendly products

- Eat food which is organic, locally-grown or in season 
- Avoid eating meat

- Buy products with less packaging

- Recycle

- Reuse or repair items instead of throwing them away

- Compost your kitchen waste

- Save water by taking shorter showers

- Turn off the tap while you brush your teeth

- Write to your representative about an environmental issue

- Take part in a protest about an environmental issue

On a scale from (strongly disagree) to (strongly agree) how do you agree with following statements:

- 'I think of myself as an environmentally-friendly consumer'

- 'I think of myself as someone who is very concerned with environmental issues'

- 'I would be embarrassed to be seen as having an environmentally friendly lifestyle'

- 'I would not want my family or friends to think of me as someone who is concerned about environmental issues'

How important is the issue of climate change to you personally?

- Very important

- Moderately important

- Slightly important

- Not at all important

Do you think climate change is something that is affecting or is going to affect you, personally?

- Yes

- No 
- I don't know

How much, if anything, would you say you know about climate change?

- A lot

- A moderate amount

- A little

- Nothing, have never heard of it

Do you think:

- Climate change is caused only by natural processes

- Climate change is caused only by human activity

- Climate change is caused by both natural processes and human activity

- There's no such thing as climate change

- I don't know what is causing climate change'

\section{Demographic Questions}

In the last page participants declared their age, gender, marital status, education level and the city in which they answered the survey.

- Gender

- Male

- Female

- Age

- Marital Status

- Single, never married

- Married/Domestic partnership

- Separated/Divorced 
- Widowed

- Education

- Some high school or less

- High school diploma

- Some college (1 yr. to less than 2 yrs.)

- Two-year college degree (A.A.)

- Four-year college degree (B.A. or B.S.)

- MA/PhD, MD, MBA, Law Degree

- Location (City)

\section{Results}

\section{Descriptive Statistics}

There were 138 subjects in the current study, out of which 89 were males and 49 females. The minimum age was 20 and the oldest person participated was 70 years old with a mean of age of 37.64 years old and a median of 33.5 years. Sixty eight subjects declared themselves as single, 58 as married, 10 as divorced and 2 as widowed. The majority said that they had a 4-year college education $(\mathrm{N}=72), 20$ individuals had a 2-year college degree, 18 had some college education but less than 2 years, 16 finished high school, 11 had post-graduate education, and one person said they hadn't finished high school. Figure 1 depicts these demographic information. 

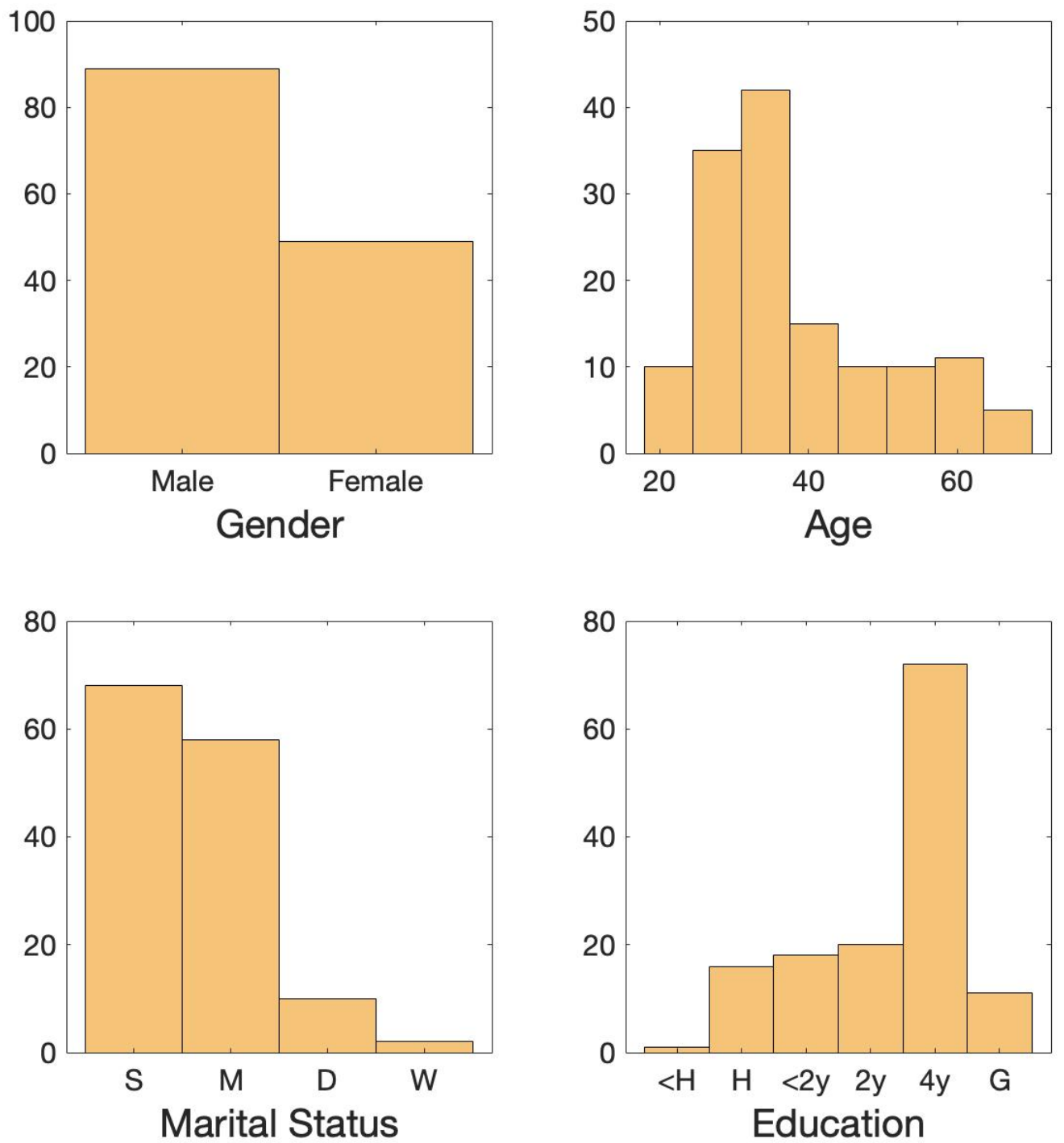

Figure 1: Histograms 

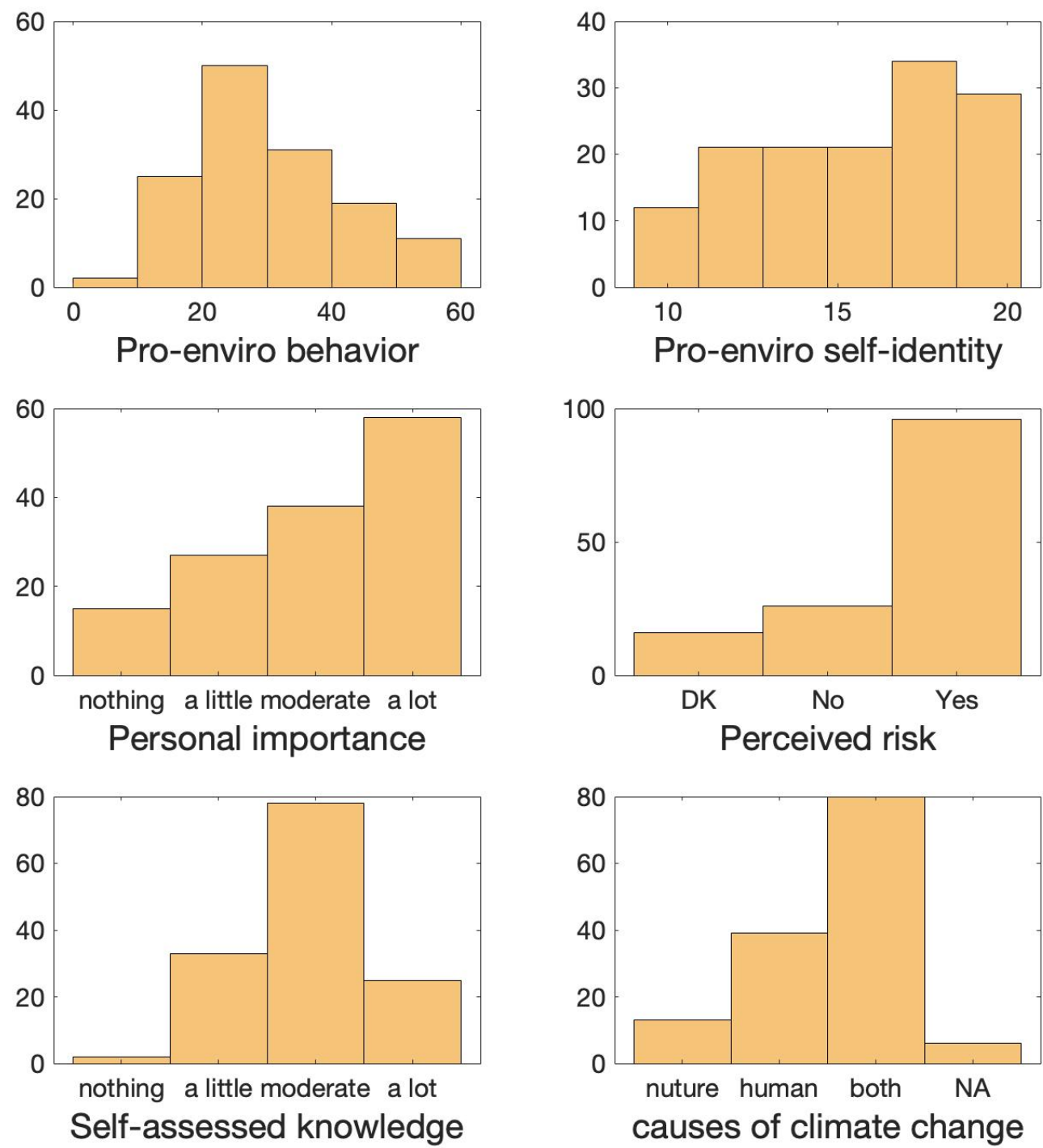

Figure 2: Histograms

\section{Pro-environmental indices}

Table 1 shows the range, mean, and standard deviation for different scales of pro-environmental features in our study. The main pro-environmental behavior index had a range of minimum of 12 to maximum of 59 in our sample with a mean of 31.80 and standard deviation of 12.14. It is also shown as a histogram in the first panel of Figure 2.

Another index was the pro-environmental self-identity in a range of 5-16 and a mean of 11.52 and SD of 3.24 (Panel 2 in Figure 2). 
Table 1: Ranges, Means and Standard Deviations for Pro-environmental indices

\begin{tabular}{lrrrr}
\hline \hline \multicolumn{1}{c}{ task parameter } & min & max & mean & \multicolumn{1}{c}{ SD } \\
\hline Pro-environmental behavior-1 & 12 & 59 & 31.80 & 12.14 \\
Pro-environmental behavior-2 & 0 & 21 & 4.52 & 5.05 \\
Pro-environmental behavior-3 & 5 & 50 & 25.03 & 8.51 \\
Pro-environmental self-identity & 0 & 3 & 2.01 & 1.03 \\
personal importance & 1 & 3 & 2.58 & 0.69 \\
self-assessed knowledge & 0 & 3 & 1.91 & 0.69 \\
\hline \hline
\end{tabular}

The personal importance index measures the subjective feeling of participants about how the prosustainability is a major theme of their mentality. This discrete measure had 4 options to answer. The majority ( $\mathrm{N}=58)$ said that it was important to them "a lot"; For 38 participants it was "moderate"; for 27 participants it mattered "a little" and for 15 participants in mattered nothing (Panel 3 in Figure 2).

In the perceived risk indie which measure how urgent global warming and other harms to the environment were felt by subjects, most of them agreed that there was a substantial risk $(\mathrm{N}=96 ;=\% 70) .26$ subjects perceived no risk and 16 subjects said they didn't know (Panel 4 in Figure 2).

In the self-assessed knowledge index, 78 participants assessed their level of knowledge to be moderate, 33 a little, 25 a lot, and 2 said they knew nothing (Panel 5 in Figure 2).

In the perceived causes for climate change, 13 participants blamed nature, 39 blamed human beings, and 80 blamed both factors (Panel 6 in Figure 2).

\section{Gender differences}

Two t-tests between two groups of gender showed that that there is no gender differences in neither the temporal discounting nor pro-environmental behavior. For the pro-environmental measure: $\mathrm{t}$-stat $=$ $-0.8146 ;$ degree of freedom $=136$; and standard deviation $=5.6735 ; \mathrm{p}=0.4167$. And for the temporal discounting scale: t-stat $=0.2710$; degree of freedom $=136$; and standard deviation $=6.0450 ; \mathrm{p}=0.7868$.

\section{Age differences}

We divided subjects roughly into two equal number groups of older and younger subjects and then ran two t-tests between these age-based groups. The results, like gender, showed that that there was no age differences in neither the temporal discounting nor pro-environmental behavior. For the proenvironmental measure: $\mathrm{t}$-stat $=-0.4649$; degree of freedom $=136$; and standard deviation $=5.6138 ; \mathrm{p}=$ 0.6428. And for the temporal discounting scale: $\mathrm{t}-\mathrm{stat}=-0.8611$; degree of freedom $=136$; and standard 
deviation $=6.0303 ; \mathrm{p}=0.3907$.

\section{Marital differences}

We also run the t-test for the two groups of single and married subjects. This time the results were significant. For the pro-environmental measure: $\mathrm{t}$-stat $=3.8881$; degree of freedom $=124$; and standard deviation $=5.52138 ; \mathrm{p}=1.6372 \mathrm{e}-04$. And for the temporal discounting scale: $\mathrm{t}$-stat $=1.7704$; degree of freedom $=124 ;$ and standard deviation $=6.0832 ; \mathrm{p}=0.0791$. (Figure 3 ).

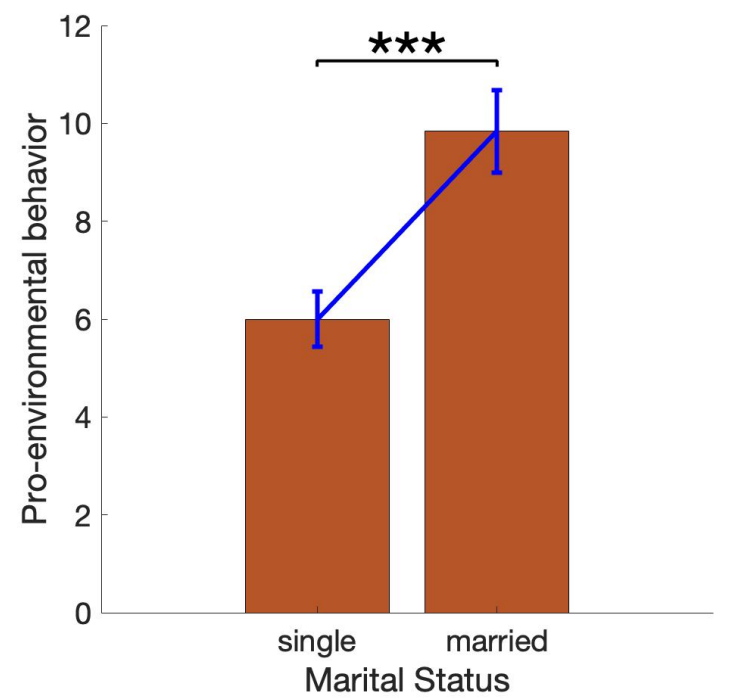

Figure 3: Pro-environmental behavior difference in single and married participants

\section{Education differences}

We also divided subjects into two groups of higher and lower educated subjects and then ran two t-tests between these age-based groups. This time also the results were significant. For the pro-environmental measure: $\mathrm{t}$-stat $=3.2072 ;$ degree of freedom $=136$; and standard deviation $=5.4838 ; \mathrm{p}=0.0017$. And for the temporal discounting scale: $\mathrm{t}$-stat $=1.1061$; degree of freedom $=136$; and standard deviation $=$ $6.0197 ; \mathrm{p}=0.2706$. (Figure 4). 


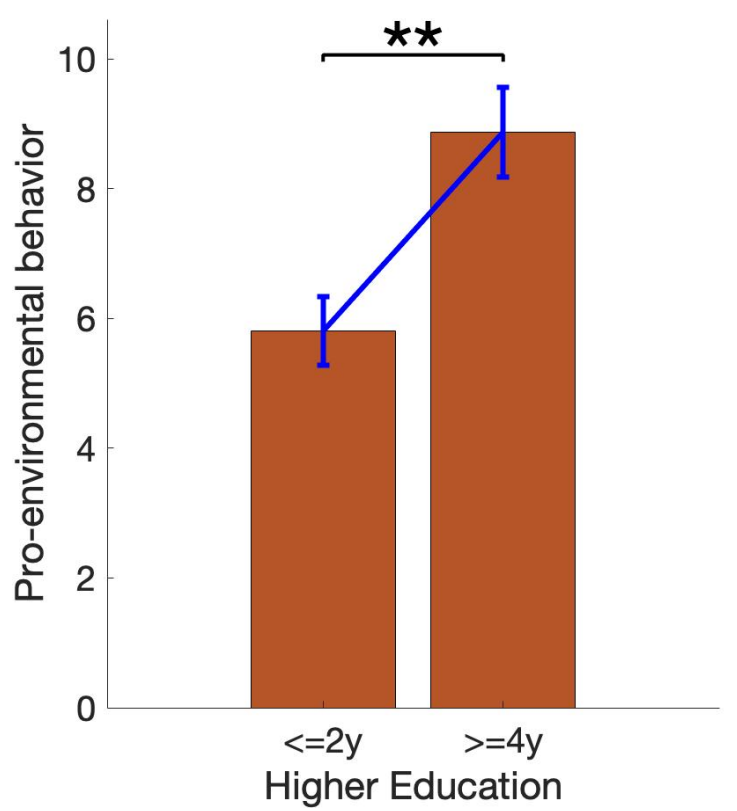

Figure 4: Pro-environmental behavior difference in participants with higher and lower Education (4-year college degree and higher versus 2-year degree and lower)

\section{Temporal Discounting measures}

For the temporal discounting measure we obtained 5 different $k$ values for each participant as a measure of how much they discount future reward. We also can simply estimate that measure just by counting the number of times participants chose the immediate versus delayed reward. Table 2 shows the range, mean and standard deviations of temporal discounting indices ( $k$ 's and \# today items) in 138 participants of our study which is similar to previous studies using the same measure [2, 36]. Figure 5 shows the histogram of distribution of temporal discounting indices in the sample $(\mathrm{N}=138)$. 
Table 2: Correlations between task parameters and temporal discounting indices

\begin{tabular}{|c|c|c|c|c|}
\hline parameter & temporal index & $\mathrm{r}$ & $\mathrm{p}$ & $\mathrm{N}$ \\
\hline \multirow[t]{6}{*}{ pro-environmental 1} & Overall k & 0.16 & 0.07 & 138 \\
\hline & Small k & 0.15 & 0.08 & 138 \\
\hline & Medium k & 0.15 & 0.09 & 138 \\
\hline & Large $\mathrm{k}$ & 0.14 & 0.09 & 138 \\
\hline & Geomean k & 0.13 & 0.09 & 138 \\
\hline & \# later items & 0.15 & 0.08 & 138 \\
\hline \multirow[t]{6}{*}{ pro-environmental 2} & Overall k & 0.28 & 0.026 & 138 \\
\hline & Small k & 0.21 & 0.053 & 138 \\
\hline & Medium k & 0.22 & 0.047 & 138 \\
\hline & Large $\mathrm{k}$ & 0.23 & 0.035 & 138 \\
\hline & Geomean k & 0.25 & 0.026 & 138 \\
\hline & \# later items & 0.28 & 0.064 & 138 \\
\hline \multirow[t]{6}{*}{ pro-environmental 3} & Overall k & 0.11 & 0.08 & 138 \\
\hline & Small k & 0.15 & 0.08 & 138 \\
\hline & Medium k & 0.13 & 0.08 & 138 \\
\hline & Large $\mathrm{k}$ & 0.12 & 0.07 & 138 \\
\hline & Geomean k & 0.11 & 0.05 & 138 \\
\hline & \# later items & 0.14 & 0.04 & 138 \\
\hline \multirow[t]{6}{*}{ self-assessed knowledge } & Overall k & -0.05 & 0.656 & 138 \\
\hline & Small k & -0.04 & 0.726 & 138 \\
\hline & Medium k & 0.02 & 0.848 & 138 \\
\hline & Large $\mathrm{k}$ & -0.04 & 0.744 & 138 \\
\hline & Geomean k & -0.02 & 0.137 & 138 \\
\hline & \# later items & 0.03 & 0.764 & 138 \\
\hline \multirow[t]{5}{*}{ self-identity } & Overall k & -0.06 & 0.46 & 138 \\
\hline & Small k & -0.08 & 0.23 & 138 \\
\hline & Medium k & -0.06 & 0.25 & 138 \\
\hline & Large $\mathrm{k}$ & -0.07 & 0.33 & 138 \\
\hline & Geomean k & -0.06 & 0.4 & 138 \\
\hline
\end{tabular}


In our research, it turned out that all of these indices are highly correlated with each other and all have very similar relationship with different pro-environmental scales. The more simple measure of \# today items has a Pearson's correlation coefficient between $.89-1$ with the more complicated $k$ measures.

\section{Correlations between pro-environmental measures and temporal discount-}

\section{ing}

Figure 5 shows the correlations between temporal discounting measures and pro-environmental behavior for all 138 participants. As we can see, there is almost a positive trend between temporal discounting and pr-environmental behavior. On the other hand there is no trend in Figure 6 which shows the correlation between temporal discounting and the pro-environmental self-identity. It seems, there is a discrepancy between behavior and attitude in current study as documented ubiquitously elsewhere in psycho-social research [17, 47,-52].

There were several questions in our survey that discriminate between participants who care versus not care about the environment. One explicit one is the following question:

- "Do you think climate change is something that is affecting or is going to affect you, personally?"

Out of 138 participants in our study, 96 of them answered "yes" to this question (= $\% 70$ of subjects). We asked whether the nature of the relationship between temporal discounting and the pro-environmental behavior changes depending on this grouping. Figure 7 shows the result of correlations between temporal discounting and pro-environmental behavior for those who answered "yes" to the above-mentioned question. As you can see, there is a significant difference and the observed trend in Figure 5 now become statistically highly significant. Pearson $r$ coefficient $=0.28 ; p=0.006$. 

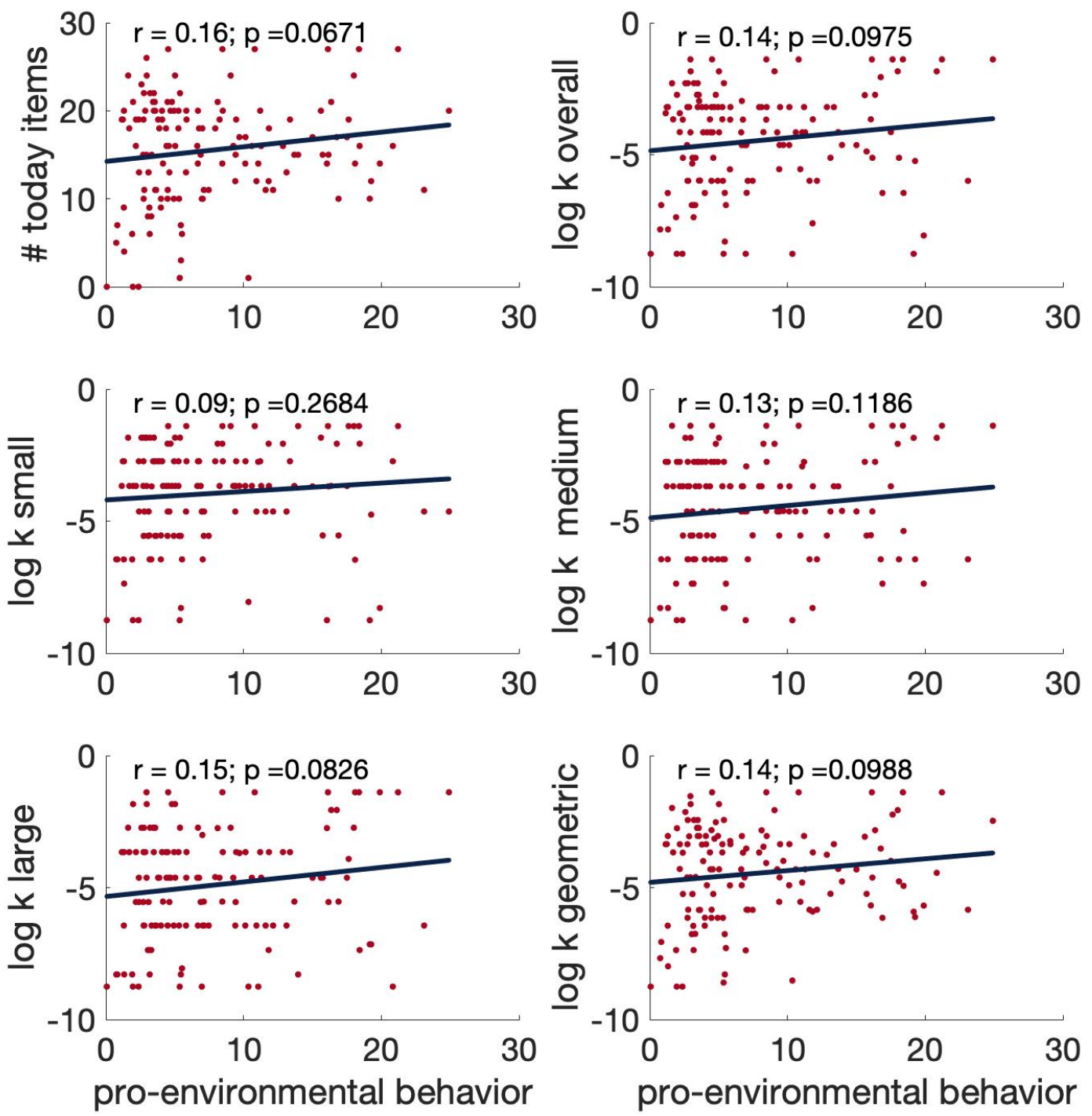

Figure 5: Correlations between temporal discounting and pro-environmental behavior scale 

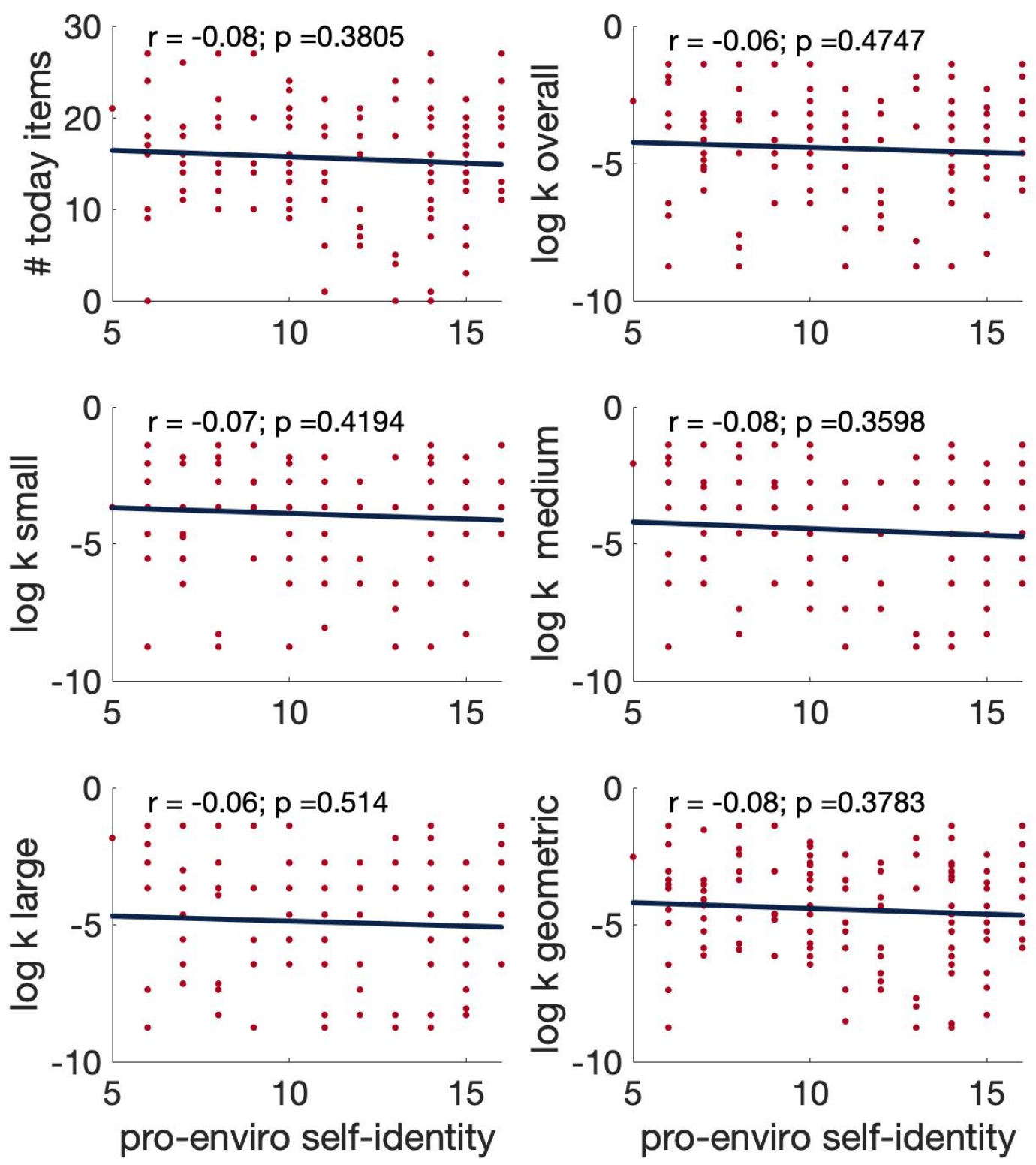

Figure 6: Correlations between temporal discounting and pro-environmental self-identity scale 

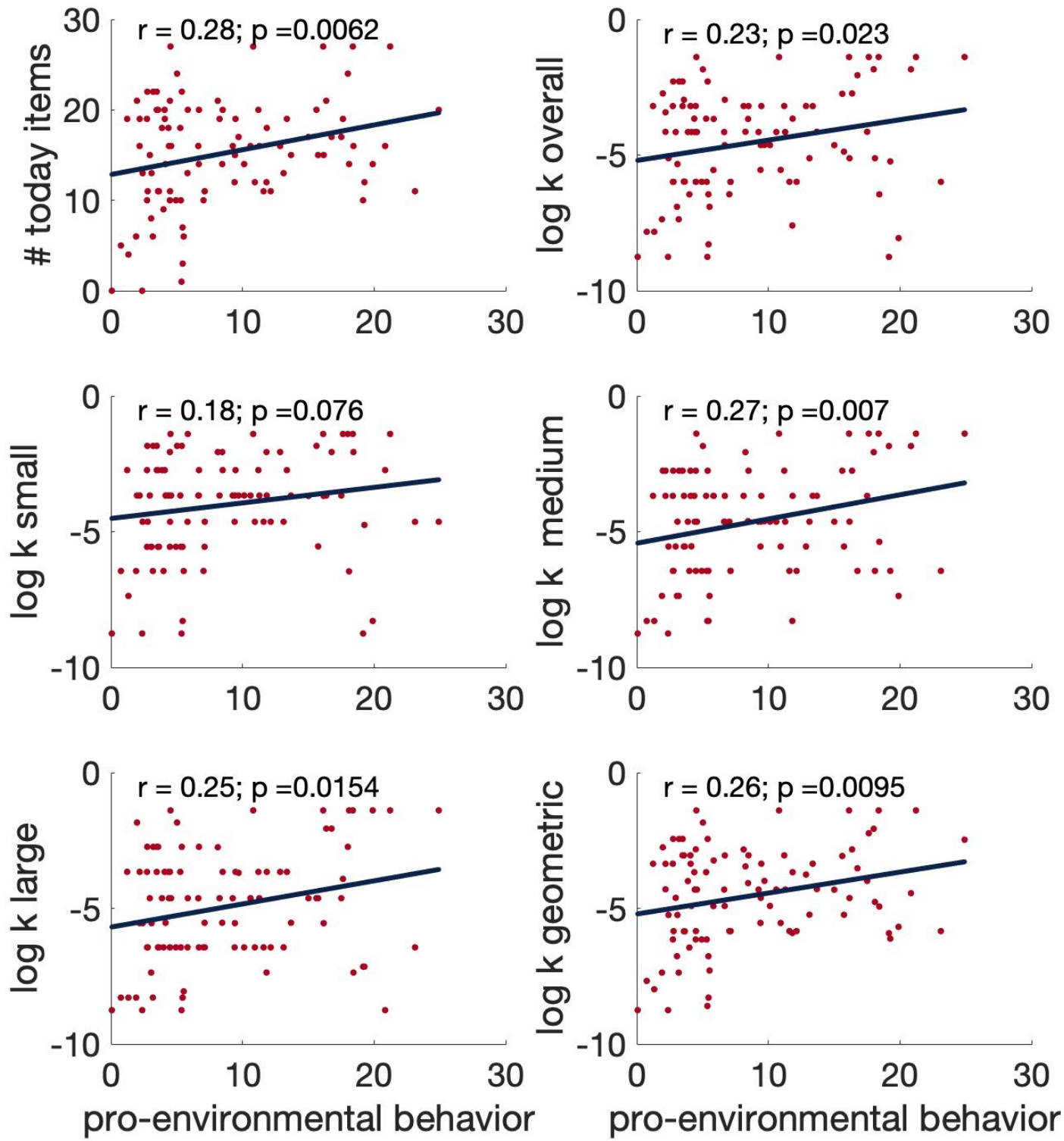

Figure 7: Correlations between temporal discounting and pro-environmental behavior scale for those participants who answered "Yes" to the question "Do you think climate change is something that is affecting or is going to affect you, personally?"

\section{Discussion}

In this study we investigated the correlation between temporal discounting measured by a monetary choice questionnaire [36] and different scales of the pro-environmental behaviors. We found a negative correlation between temporal discounting and pro-environmental behavior measure. This relationship was much more stronger if we filter out $\% 30$ of subjects who explicitly declared that they didn't care 
about this issue at all. We found no correlation between temporal discounting and pro-environmental self-identity which was unexpected to us. Although we found marriage and education both strengthen pro-environmental behaviors.

There are several limitations in the current study. First, the chosen measures for both temporal discounting and pro-environmental behaviors are very specific. This fact would harm the generalizibility of our results. Although a strong correlation between different measures of temporal discounting has been demonstrated in several studies [53, 54], most of these measures are monetary which may have weak relationships with delay discounting in other domains [55]. Pro-environmental behaviors also have been studied in many different and diverse settings including natural settings, virtual realities, hypothetical stories, repeated choice and sequential choice paradigms and it seems there is no single shared factor underlying a core pro-environmental tendency in all of these settings. Only way to find out how generlizable our results are is to replicate it using other measures of pro-environmental and temporal discounting. This will provide us with more evidence to support or reject the preliminary results and assess the generlizablilty of the current results.

Another important limitation of our study is recruiting participants online through MTurk. This setting obviously is not ideal and there is no control over the atmosphere in which subjects participate in study. The quality of data is under question in this modality.

Lastly, we need to hypothesise about the mediating roles of other factors between temporal discounting trait and pro-environmental behavior. How exactly a discounting attitude towards future would translate into a pro-environmental action? Future research should bring more variables within the game to better illuminate the mechanisms behind pro-environmental behaviors.

\section{References}

1. Whitmarsh, L. \& O'Neill, S. Green identity, green living? The role of pro-environmental self-identity in determining consistency across diverse pro-environmental behaviours. Journal of Environmental Psychology 30, 305-314. ISSN: 02724944 (2010).

2. Kirby, K. N. \& Maraković, N. N. Delay-discounting probabilistic rewards: Rates decrease as amounts increase. Psychonomic Bulletin and Review 3, 100-104. ISSN: 10699384 (Mar. 1996). 
3. WCED, S. W. S. World Commission On Environment And Development. Our common future 17, 1-91 (1987).

4. Hirsh, J. L., Costello, M. S. \& Fuqua, R. W. Analysis of delay discounting as a psychological measure of sustainable behavior. Behavior and Social Issues 202, 187-202 (2015).

5. Sara, A. Pro-Environmental Behavior and Its Antecedents as a Case of Social and Temporal Dilemmas. British Journal of Education, Society \& Behavioural Science 4, 508-526 (2014).

6. Lange, F. \& Dewitte, S. Measuring pro-environmental behavior: Review and recommendations 2019.

7. Sparks, P. \& Shepherd, R. Self-identity and the theory of planned behaviour: assessing the role of identification with green consumerism. Social Psychology Quarterly 55, 388-399 (1992).

8. Arbuthnott, K. D. Taking the long view: Environmental sustainability and delay of gratification. Analyses of Social Issues and Public Policy 10, 4-22. ISSN: 15297489 (2010).

9. Sadeghiyeh, H., Khanahmadi, I., Farhadbeigi, P. \& Karimi, N. Cognitive reflection and the coronavirus conspiracy beliefs. PsyArXiv (2020).

10. Wittmann, M. \& Sircova, A. Dispositional orientation to the present and future and its role in pro-environmental behavior and sustainability. Heliyon 4, e00882. ISSN: 24058440. https://doi.org/10.1016/j.heliyon.2018.e00882(2018).

11. Arnocky, S., Milfont, T. L. \& Nicol, J. R. Time perspective and sustainable behavior: Evidence for the distinction between consideration of immediate and future consequences. Environment and Behavior 46, 556-582 (2014).

12. Bamberg, S. \& Schmidt, P. Incentives, morality, or habit? Predicting students' car use for university routes with the models of Ajzen, Schwartz, and Triandis. Environment and behavior 35, 264-285 (2003).

13. Jackson, T. Motivating sustainable consumption: A review of evidence on consumer behaviour and behavioural change. Sustainable development research network 29, 30-40 (2005). 
14. Jackson, J. J. M., Procidano, M. M. E. \& Cohen, C. J. C. Subject pool sign-up procedures: a threat to external validity. Social Behavior and Personality: an international journal 17, 29-42. ISSN: 03012212 (Jan. 1989).

15. Sadeghiyeh, H., Khorrami, A. \& Hatami, J. Gender differences in sensorimotor empathy for pain: a single-pulse TMS study. Curr. Neurobiol 8, 99-111 (2017).

16. Kollmuss, A. \& Agyeman, J. Mind the gap: why do people act environ- mentally and what are the barriers to pro-environmental behavior. Environmental education research 8, 239260 (2002).

17. Newhouse, N. Implications of attitude and behavior research for environmental conservation. The Journal of Environmental Education, 22, 26-32 (1990).

18. Heward, W. L. \& Kimball, J. W. Sustaining sustainability with clueless contingencies. Sustain: A Journal of Environmental and Sustainability Issues 28, 4-15 (2013).

19. Luke, M. M. \& Alavosius, M. Impacting community sustainability through behavior change: A research framework. Behavior and Social Issues 21, 54-79 (2012).

20. Whitmarsh, L. \& O’Neill, S. Green identity, green living? The role of pro-environmental self-identity in determining consistency across diverse pro-environmental behaviours. Journal of environmental psychology 30, 305-314 (2010).

21. Stern, P. Toward a coherent theory of environmentally significant behavior. Journal of Social Issues 56, 407-424 (2000).

22. Whitmarsh, L. Behavioural responses to climate change: asymmetry of intentions and impacts. Journal of Environmental Psychology 29, 13-23 (2009).

23. Whitmarsh, L., O’Neill, S., Seyfang, G. \& Lorenzoni, I. Carbon capability: What does it mean, how prevalent is it, and how can we promote it? Tyndall Working Paper 132 (2009).

24. Kaplan, B. A. et al. Automating Scoring of Delay Discounting for the 21- and 27-Item Monetary Choice Questionnaires. Behavior Analyst 39, 293-304. ISSN: 21968918 (Oct. 2016). 
25. Hardin, G. The tragedy of the commons: the population problem has no technical solution; it requires a fundamental extension in morality. Science 162, 1243-1248 (1968).

26. Berry, M. S., Nickerson, N. P. \& Odum, A. L. Delay discounting as an index of sustainable behavior: Devaluation of future air quality and implications for public health. International Journal of Environmental Research and Public Health 14, 1-14. ISSN: 16604601 (2017).

27. Daugherty, J. R. \& Brase, G. L. Taking time to be healthy: Predicting health behaviors with delay discounting and time perspective. Personality and Individual differences $\mathbf{4 8 ,}$ 202-207 (2010).

28. Lagorio, C. H. \& Madden, G. J. Delay discounting of real and hypothetical rewards III: Steady-state assessments, forced-choice trials, and all real rewards. Behavioural Processes 69, 173-187 (2005).

29. Sadeghiyeh, H. Electronic Supplementary Material 1. Journal of Individual Differences $\mathbf{0}$, $1-11(2020)$.

30. Madden, G., Bickel, W. K. \& Jacobs, E. A. Discounting of delayed rewards in opioiddependent outpatients: exponential or hyperbolic discounting functions? Experimental and clinical psychopharmacology 7, 284-93 (Aug. 1999).

31. Reynolds, B., Richards, J. B., Horn, K. \& Karraker, K. Delay discounting and probability discounting as related to cigarette smoking status in adults. Behavioural Processes 65, 35-42. ISSN: 03766357 (2004).

32. Ståhl, T. \& van Prooijen, J. W. Epistemic rationality: Skepticism toward unfounded beliefs requires sufficient cognitive ability and motivation to be rational. Personality and Individual Differences 122, 155-163. ISSN: 01918869 (2018).

33. Sadeghiyeh, H. et al. On the psychology of the psychology subject pool : an exploratory test of the good student effect. Journal of Individual Differences, 1-11 (2020).

34. Berinsky, A. J., Huber, G. A. \& Lenz, G. S. Evaluating online labor markets for experimental research: Amazon.com's mechanical turk. Political Analysis 20, 351-368. ISSN: 10471987 (2012). 
35. Casler, K., Bickel, L. \& Hackett, E. Separate but equal? A comparison of participants and data gathered via Amazon's MTurk, social media, and face-to-face behavioral testing. Computers in Human Behavior 29, 2156-2160. ISSN: 07475632. http : / / dx . doi . org/10.1016/j.chb.2013.05.009(2013).

36. Kirby, K. N., Petry, N. M. \& Bickel, W. K. Heroin addicts have higher discount rates for delayed rewards than non-drug-using controls. Journal of Experimental Psychology: General 128, 78-87. ISSN: 00963445 (1999).

37. Sadeghiyeh, H. et al. Temporal discounting correlates with directed exploration but not with random exploration. Scientific Reports 10. ISSN: 20452322 (2020).

38. Green, L., Fry, A. F. \& Myerson, J. Discounting of delayed rewards: A Life-Span Comparison. Psychological Science 5, 33-36. ISSN: 14679280 (1994).

39. Sadeghiyeh, H. Social Influence, Temporal Discounting and Active-Passive Gap in ExploreExploit Dilemma PhD thesis (University of Arizona, 2019), 61.

40. Mazur, J. E. in Quantitative analyses of behavior: vol. 5. The effect of delay and of intervening events on reinforcement value (eds Commons, M. L., Mazur, J. E. \& Nevin, J. A.) 55-73 (1987). ISBN: 0-89859-800-1 (Hardcover).

41. Sadeghiyeh, H., Wang, S. \& Wilson, R. C. Lessons from a "failed" replication: The importance of taking action in exploration. PsyArXiv (2018).

42. Kaplan, B. A., Lemley, S. M., Reed, D. D. \& Jarmolowicz, D. P. 21 - and 27-Item Monetary Choice Questionnaire Automated Scorer [spreadsheet application] 2014. https: //kuscholarworks.ku.edu/handle/1808/15424.

43. Markle, G. L. Pro-Environmental Behavior: Does It Matter How It's Measured? Development and Validation of the Pro-Environmental Behavior Scale (PEBS). Human Ecology 41, 905-914. ISSN: 03007839 (2013).

44. Cook, A. J., Kerr, G. N. \& Moore, K. Attitudes and intentions towards purchasing GM food. Journal of Economic Psychology, 23, 557-572 (2002).

45. DEFRA. A framework for pro-environmental behaviours. London: Depart- ment for Environment, Food and Rural Affairs. DEFRA. (0). 
46. Sadeghiyeh, H., Khorrami, A. \& Hatami, J. Gender differences in empathy for pain: A single-pulse transcranial magnetic stimulation study. Neurophysiologie Clinique/Clinical Neurophysiology 42, 62 (2012).

47. HASHEM, S., NASER, A. \& ANAHITA, K. B. Comparing Situational and Dispositional Empathy, Considering the role of gender. Social Psychology Research 1, 1-16 (2011).

48. Ajzen, I., Brown, T. C. \& Carvajal, F. Explaining the discrepancy between intentions and actions: The case of hypothetical bias in contingent valuation. Personality and Social Psychology Bulletin 30, 1108-1121. ISSN: 01461672 (Sept. 2004).

49. Gross, S. J. \& Niman, C. M. Attitude-behavior consistency: A review. Public opinion quarterly 39, 358-368 (1975).

50. Armitage, C. J. \& Christian, J. From attitudes to behaviour: Basic and applied research on the theory of planned behaviour. Current psychology 22, 187-195 (2003).

51. Sadeghiye, H., Aghababaei, N., Hatami, J. \& Bonaraki, A. K. Comparing situational and dispositional empathy, considering the role of gender. Social Psychology Research Summer, 1-16 (2011).

52. Rumph, R., Ninness, C., McCuller, G. \& Ninness, S. K. Guest editorial: Twenty years later, commentary on Skinner's" Why we are not acting to save the world". Behavior and Social Issues14 14, 1 (2005).

53. Basile, A. G. \& Toplak, M. E. Four converging measures of temporal discounting and their relationships with intelligence, executive functions, thinking dispositions, and behavioral outcomes. Frontiers in Psychology 6. ISSN: 16641078 (2015).

54. Epstein, L. H. et al. Comparison between two measures of delay discounting in smokers. Experimental and Clinical Psychopharmacology 11, 131-138. ISSN: 10641297 (May 2003).

55. Weatherly, J. N., Terrell, H. K. \& Derenne, A. Delay discounting of different commodities. Journal of General Psychology 137, 273-286. ISSN: 00221309 (2010). 
56. Sadeghiyeh, H. (2019). Social influence, temporal discounting and active-passive gap in explore-exploit dilemma (Doctoral dissertation). Tucson, AZ: University of Arizona. 